\title{
Endoscopic Transection for Intramural Esophageal Dissection Caused by a Retropharyngeal Abscess
}

\author{
Gwang Ha Kim ${ }^{1,2}$, Yeong Dae Kim²,3 \\ Department of Internal Medicine, Pusan National University School of Medicine ${ }^{1}$, Biomedical Research Institute, Pusan National University \\ Hospital $^{2}$, Department of Thoracic and Cardiovascular Surgery, Pusan National University School of Medicine ${ }^{3}$, Busan, Korea
}

Question: A 66-year-old man was admitted with severe pharyngeal pain, dysphagia and dyspnea. Laryngoscopy revealed severe laryngeal edema (Fig. 1A), and neck CT showed a non-collecting retropharyngeal abscess (Fig. 1B). He underwent tracheostomy, and despite antibiotic therapy, his fever and chills persisted with worsening dysphagia. Endoscopy showed diffuse swelling between the upper esophagus and the gastric cardia and an upper esophageal opening discharging pus (Fig. 2A, B). EUS revealed that the swelling of the esophagus and the gastric cardia was caused
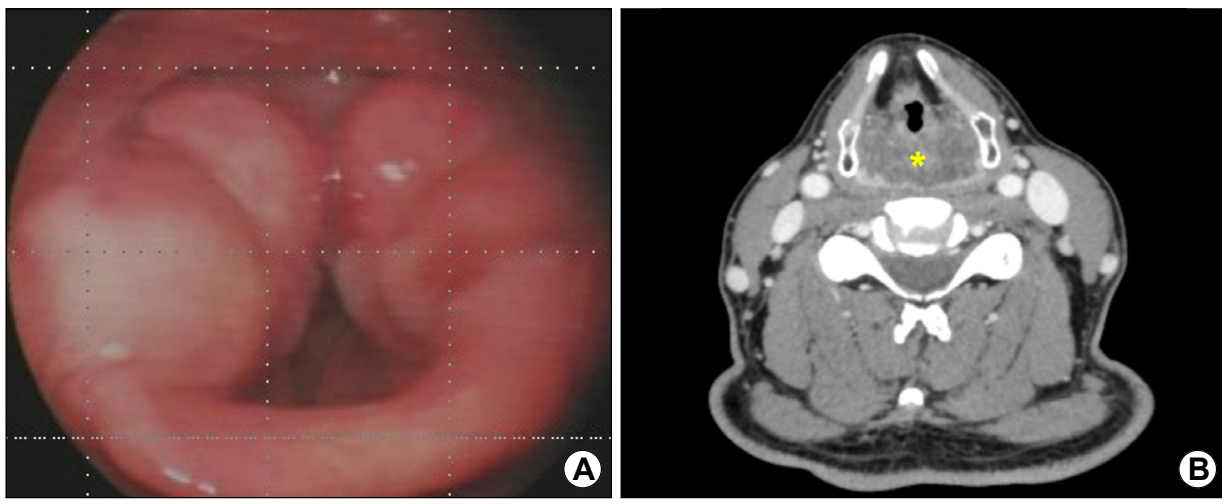

Fig. 1. (A) Laryngoscopy shows severe laryngeal edema. (B) Neck CT scan shows a non-collecting retropharyngeal abscess (asterisk).
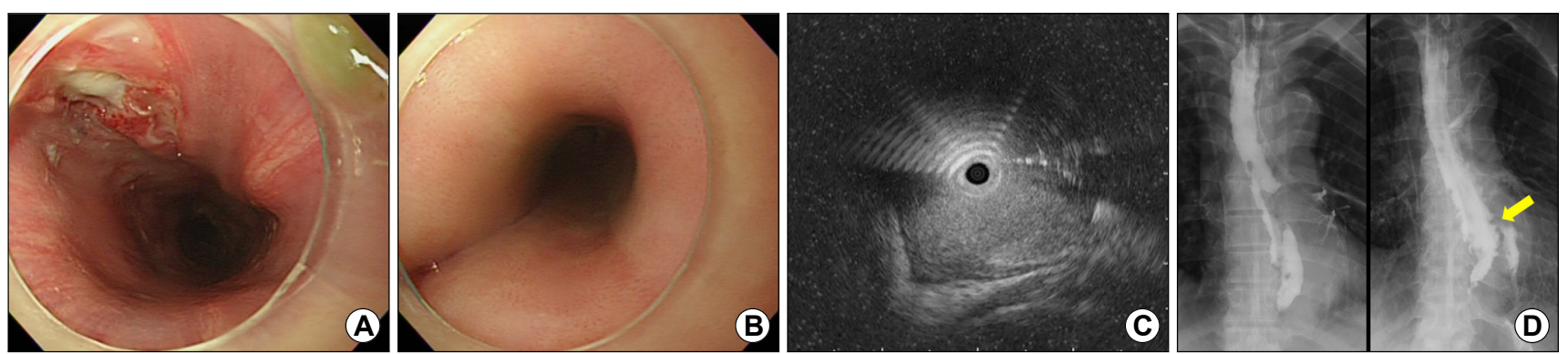

Fig. 2. (A, B) Endoscopy reveals diffuse swelling extending between the upper esophagus and gastric cardia, as well as an upper esophageal opening discharging pus. (C) EUS reveals that the swelling of the esophagus and gastric cardia is caused by hyperechoic floating material in the submucosal layer, suggesting submucosal pus accumulation. (D) Esophagography shows esophageal dissection extending between the upper and lower esophagus, with a small amount of leakage from the false lumen of the lower esophagus (arrow).

Received: August 4, 2019 Revised: August 16, 2019 Accepted: August 17, 2019

Corresponding author: Gwang Ha Kim

Department of Internal Medicine, Pusan National University School of Medicine, and Biomedical Research Institute, Pusan National University Hospital, 179 Gudeok-ro, Seo-gu, Busan 49241, Korea

Tel: +82-51-240-7869, Fax: +82-51-244-8180, E-mail: doc0224@pusan.ac.kr

Copyright $\odot 2020$ Korean College of Helicobacter and Upper Gastrointestinal Research

(a) The Korean Journal of Helicobacter and Upper Gastrointestinal Research is an Open-Access Journal. All articles are distributed under the terms of the Creative Commons Attribution Non-Commercial License (http:// creativecommons.org/licenses/by-nc/4.0) which permits unrestricted non-commercial use, distribution, and reproduction in any medium, provided the original work is properly cited. 

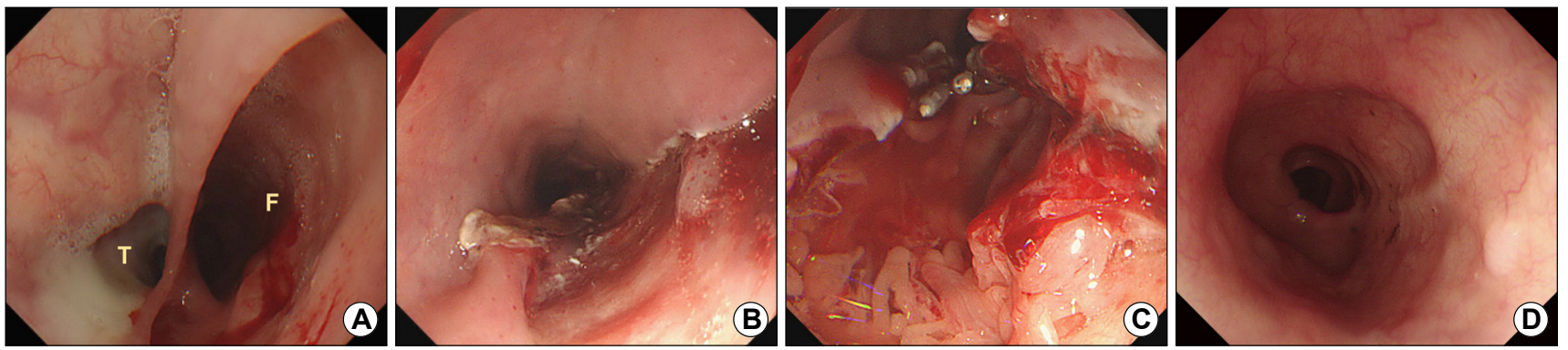

Fig. 3. (A, B) Endoscopic transection is performed for the mucosal bridge between the true $(\mathrm{T})$ and false $(\mathrm{F})$ esophageal lumens from the upper to lower esophagus approximately $2 \mathrm{~cm}$ proximal to the esophagogastric junction. (C) Endoscopic clipping is performed for a suspected leakage site. (D) Follow-up endoscopy shows a single esophageal lumen.

by submucosal pus accumulation (Fig. 2C), and esophagography showed esophageal dissection between the upper and lower esophagus with a small amount of leakage from the false lumen of the lower esophagus (Fig. 2D).

What is the appropriate treatment?

Answer: Using a hook-knife (Olympus, Tokyo, Japan), the mucosal bridge between the true and false lumens from the upper to lower esophagus approximately $2 \mathrm{~cm}$ proximal to the esophagogastric junction was endoscopically transected; then, endoscopic clipping was performed for a suspected leakage site (Fig. $3 \mathrm{~A} \sim \mathrm{C}$ and Video 1). Oral intake was resumed, and the patient was discharged 2 weeks later after decannulation. Follow-up endoscopy 2 months later showed a single esophageal lumen (Fig. 3D). The patient was asymptomatic at the 2-year follow-up.

Intramural esophageal dissection is rare and characterized by sudden severe retrosternal pain, hematemesis, odynophagia, and dysphagia secondary to longitudinal separation of the submucosa from the esophageal muscularis propria. ${ }^{1}$ It is usually precipitated by a rapid increase in intraesophageal pressure such as intramural hematoma formation. ${ }^{2}$ Conservative management usually produces favorable outcomes. ${ }^{3}$ Our patient showed a retropharyngeal abscess that progressed to intramural esophageal dissection and persistent infection and dysphagia despite conservative management; however, he was successfully treated endoscopically.
Video 1. Endoscopic transection of the mucosal bridge between the true and false esophageal lumens.

\section{CONFLICT OF INTEREST}

No potential conflict of interest relevant to this article was reported.

\section{ORCID}

Gwang Ha Kim (10) https://orcid.org/0000-0001-9721-5734

Yeong Dae Kim (1) https://orcid.org/0000-0001-7135-4648

\section{REFERENCES}

1. Gluck M, Jiranek GC, Low DE, Kozarek RA. Spontaneous intramural rupture of the esophagus: clinical presentation and endoscopic findings. Gastrointest Endosc 2002;56:134-136.

2. Mathieu B, Le Gall P, Mourani A, Marquand A, Schaefer JP. So-called spontaneous intramural dissection of the esophagus. Gastroenterol Clin Biol 2000;24:1117-1121.

3. Benatta MA, Grimaud JC, Kaci M, Desjeux A, Baghdadi M, Loumi A. Intramural esophageal dissection due to pharyngeal abscess treated by endoscopic esophageal transection: a case report. Gastroenterol Clin Biol 2010;34:329-331. 